\title{
INTEGRAL-BASED PARAMETER IDENTIFICATION FOR LONG-TERM DYNAMIC VERIFICATION OF A GLUCOSE-INSULIN SYSTEM MODEL
}

\author{
Christopher E. Hann ${ }^{1}$, J Geoffrey Chase ${ }^{1}$, Jessica Lin ${ }^{1}$, Thomas Lotz ${ }^{1}$, Carmen V \\ Doran $^{1}$, and Geoffrey M Shaw ${ }^{2}$
}

${ }^{1}$ Department of Mechanical Engineering, Centre for Bio-Engineering, University of Canterbury, Private Bag 4800, Christchurch, New Zealand, Email:

geoff.chase@canterbury.ac.nz

${ }^{2}$ Department of Intensive Care, Christchurch Hospital, Christchurch School of

Medicine and Health Science, University of Otago, Christchurch, New Zealand

\begin{abstract}
Hyperglycaemia in critically ill patients increases the risk of further complications and mortality. This paper introduces a model capable of capturing the essential glucose and insulin kinetics in patients from retrospective data gathered in an Intensive Care Unit (ICU). The model uses two time-varying patient specific parameters for glucose effectiveness and insulin sensitivity. The model is mathematically reformulated in terms of integrals to enable a novel method for identification of patient specific parameters. The method was tested on long-term blood glucose recordings from 17 ICU patients, producing 4\% average error, which is within the sensor error. One-hour forward predictions of blood glucose data proved acceptable with an error of $2-11 \%$. All identified parameter values were within reported physiological ranges. The parameter identification method is more accurate and significantly faster computationally than commonly used non-linear, non-convex methods. These results verify the model's ability to capture long-term observed glucose-insulin dynamics in hyperglycemic ICU patients, as well as the fitting method developed. Applications of the model and parameter identification method for automated control of blood glucose and medical decision support are discussed.
\end{abstract}


Keywords - Critical Care, Glucose, Hyperglycemia, Insulin, Modeling, Integrals, Parameter Identification

\section{INTRODUCTION}

Critically ill patients often experience stress-induced hyperglycemia and high levels of insulin resistance, even if they have no history of diabetes [1-4]. The metabolic response to stress is characterised by major changes in glucose metabolism. Increased secretion of counter-regulatory hormones leads to a prominent rise in endogenously produced glucose and the rate of hepatic gluconeogenesis, and a reduction in insulin sensitivity. Nutritional support regimes with a high carbohydrate content often compound the counter-regulatory response and do not suppress endogenous glucose production as normal $[3,4]$.

Inhibiting the response to increased glycemic levels are factors such as increased insulin resistance, absolute or relative insulin deficiency, and drug therapy. Although hyperglycemia can be a marker of severity of illness, it can also worsen outcomes, leading to an increased risk of further complications such as severe infection [5], myocardial infarctions [1], polyneuropathy and multiple-organ failure [2]. Tight glucose control has been shown to reduce ICU patient mortality by as much as $45 \%$ $[2,6]$.

To better control glucose levels, model-based [e.g. 7] and sliding-scale protocols [e.g. 8] have been tested to control glucose levels. Model-based methods can be very accurate, but require the ability to identify patient specific parameters and capture all 
of the observed dynamics. Current parameter identification methods are non-linear, non-convex and in some cases too computationally intense for real-time use in most clinical environments [9]. This paper mathematically reformulates a glucose-insulin system model for critical care patients in terms of integrals. As a result, identifying time varying patient specific parameters becomes linear and convex with minimal computation required.

A fast, accurate identification method is important in the process of refining and testing a model as many more patients can be studied in a short time. For non-linear or non-convex methods the error can be due to the model not capturing dynamics or finding a local rather than the global minima. Using many different starting points may find a better solution at the cost of increased computational time. However, there is still only a probability of finding the global minimum rather than the certainty of a convex method. More importantly, a fast, accurate convex method enables application in real-time model-based control and medical decision support applications.

\section{METHODOLOGY}

\section{A. Physiology and System Model}

The basis for the glucose-insulin system model presented is the Minimal Model by Bergman et al [10]. An addition to this model is required that accounts for un-utilised insulin in the plasma or insulin that had bound and then unbound to cell walls, tissues or insulin receptors. This addition has a similar effect to splitting the insulin compartment into a slow path and a fast path, which indicates the existence of fast and slow absorption channels and the presence of local insulin degradation [11-13]. 
Turnheim and Waldhausl [12] studied the pharmacokinetic modelling of intravenous insulin injection, and concluded that the concentration of plasma insulin following a bolus injection declines with at least two exponentials or two different rates. The first is a rapidly disappearing component of insulin representing elimination from the intravascular space, and the second is a more slowly disappearing component that reflects elimination from the interstitial fluid and the tissues that utilise insulin. These two components have half-lives of 2.4 and 50-130 minutes, respectively.

During the 1970's and 1980's, several papers were published regarding the plasma insulin disappearance kinetics in humans. Many found flaws in the first order (linear) assumptions of insulin disappearance that had been predominantly used in previous models (e.g. $[11,14])$. These flaws were assumed to be based on the narrow range of insulin levels studied, with the first reported non-proportionality between plasma concentration and plasma disappearance rate published by Sönksen et al [15]. Other experimental studies were undertaken considering the concentrations resulting from a series of intravenous insulin infusions at different rates in both normal and diabetic subjects as reviewed in [16]. These studies concluded that insulin disappearance is often governed by Michaelis-Menten saturation dynamics.

In addition, insulin mediated glucose clearance is controlled primarily by insulin sensitivity which links insulin concentration and glucose levels. As the dose of exogenous insulin is increased in controlled hyperglycemic clamp studies, insulin sensitivity decreased [17]. This result occurs because the effect of insulin saturates at 
the receptor, limiting utilisation [18]. Hence, there is a need for a saturable mechanism on insulin action. Natali et al [18] added Michaelis-menten saturation of insulin action on fractional glucose extraction in a circulatory model, and obtained good fits with limitations only occurring in the first 60 minutes, which was attributed to an irregular onset of insulin action during this initial phase. Finally, to reduce model complexity and better match known physiological response, a term must be included to suppress endogenous insulin secretion during periods of high exogenous insulin infusion [19] that are often encountered in critical care [e.g. 2,7].

The resulting model is defined:

$$
\begin{gathered}
\dot{G}=-p_{G} G-S_{I}\left(G+G_{E}\right) \frac{Q}{1+\alpha_{G} Q}+P(t) \\
Q=k \int_{0}^{t} I(\tau) e^{-k(t-\tau)} d \tau \\
\dot{I}=-\frac{n I}{1+\alpha_{I} I}+\frac{u(t)}{V_{I}}+e^{-u(t)} I_{B}
\end{gathered}
$$

where $G(t)=$ concentration of the plasma glucose above the equilibrium level $(\mathrm{mmol} / \mathrm{L}), G_{E}=$ equilibrium level of plasma glucose concentration $(\mathrm{mmol} / \mathrm{L}), I(t)=$ concentration of the plasma insulin above basal level $(\mathrm{mU} / \mathrm{L}), I_{B}=$ basal level of plasma insulin concentration $(\mathrm{mU} / \mathrm{L}), P(t)=$ exogenous glucose infusion rate $(\mathrm{mmol} /(\mathrm{L} \cdot \mathrm{min})), u(t)=$ insulin infusion rate $(\mathrm{mU} / \mathrm{min}), V_{I}=$ assumed insulin distribution volume $(\mathrm{L}), n=$ delay in interstitial transfer of insulin $\left(\min ^{-1}\right), p_{G}=$ timevarying fractional clearance of plasma glucose at basal insulin $\left(\mathrm{min}^{-1}\right), S_{I}=$ timevarying insulin sensitivity $(\mathrm{L} / \mathrm{mU} \cdot \mathrm{min}), k=$ parameter controlling the effective half 
life of insulin $\left(\mathrm{min}^{-1}\right), \alpha_{I}=$ Michaelis-Menten parameter for insulin transport saturation, $\alpha_{G}=$ Michaelis-Menten parameter for glucose clearance saturation.

The model has been developed to account for a non-linear saturation of exogenous insulin disappearance rate from plasma (in Equation (3)) and its saturable utilisation to reduce blood glucose levels (in Equation (1)). The addition of transient insulin kinetics via a convolution integral accounts for the accumulation dynamic seen in prior clinical trials [7], and better matches physiological knowledge. This model therefore effectively splits the glucose compartment into fast and slow (or available and unavailable) compartments over a continuum rather than discrete states (e.g. [20]). During periods of significant exogenous insulin infusions, the endogenous insulin production is effectively removed from the model, reducing model complexity and matching accepted assumptions for the suppression of endogenous insulin production by exogenous infusion [19].

\section{B. Parameter Fitting and Identification}

The parameters $\left(V_{I}=12 \mathrm{~L}, n=0.16 \mathrm{~min}^{-1}, k=0.0099 \mathrm{~min}^{-1}, \alpha_{G}=0.04 \mathrm{~L} / \mathrm{mU}, \alpha_{I}\right.$ $=0.0017 \mathrm{~L} / \mathrm{mU}$ ) were found through an extensive literature search [21], and are assumed to be constant and set to mean values (e.g. [12,16-18]). The exogenous feed details, $P(t)$, are known for each patient in this study. The equilibrium glucose level, $G_{E}$, can be estimated by averaging the glucose readings across the prior 8-12 hours. The reported parameter ranges are: $0.0005 \leq \alpha_{I} \leq 0.0043,0.0053 \leq k \leq 0.0139$, $0.02 \leq n \leq 0.3$ (the majority have $0.02 \leq n \leq 0.16$ ) and $0.001 \leq \alpha_{G} \leq 0.04$ [21] 
The approach is to fix $k, n, \alpha_{G}$ and $\alpha_{I}$ at average population values for all patients then identify $S_{I}$ and $p_{G}$ as time varying values. This approach can be thought of as a minimal approach where the major dynamics are identified first $\left(S_{I}\right.$ and $\left.p_{G}\right)$ before secondary parameters are modified to better fit the data if required. It is therefore important to ensure the fitting method for identifying time varying patient specific parameters is as low in computation as possible, so other parameters can be varied without significantly affecting the overall computation time. Computational time is a significant factor to consider in real time control, as well as in the process of refining and testing the model on large numbers of patients.

Integrating both sides of Equation (1) and defining $\bar{Q}=Q /\left(1+\alpha_{G} Q\right)$, the following expression holds for any segment of time from $t_{0}$ to $t$ :

$$
\begin{gathered}
\int_{t_{0}}^{t} \dot{G} d t=\int_{t_{0}}^{t}\left(-p_{G} G-S_{I}\left(G+G_{E}\right) \bar{Q}+P\right) d t \\
\Rightarrow G(t)-G\left(t_{0}\right)=-\int_{t_{0}}^{t} p_{G} G d t-\int_{t_{0}}^{t} S_{I}\left(G+G_{E}\right) \bar{Q} d t+\int_{t_{0}}^{t} P d t
\end{gathered}
$$

Substituting the total glucose level $G_{T}=\left(G+G_{E}\right)$ into Equation (4) results in an equivalent expression that is easy to compute given measured total glucose levels:

$$
G_{T}(t)-G_{T}\left(t_{0}\right)=-\int_{t_{0}}^{t} p_{G}\left(G_{T}-G_{E}\right) d t-\int_{t_{0}}^{t} S_{I} G_{T} \bar{Q} d t+\int_{t_{0}}^{t} P d t
$$


To reduce computational complexity and account for a variation over time, the total time interval is divided into equal segments during which $p_{G}$ and $S_{I}$ are defined as piecewise constant.

$$
\begin{aligned}
& p_{G}=\sum_{i=1}^{N} p_{G i}\left(H\left(t-t_{(i-1)}\right)-H\left(t-t_{i}\right)\right) \\
& S_{I}=\sum_{i=1}^{N} S_{I i}\left(H\left(t-t_{(i-1)}\right)-H\left(t-t_{i}\right)\right)
\end{aligned}
$$

where $H\left(t-t_{0}\right)$ is the Heaviside function defined $H\left(t-t_{0}\right)=0$ when $t$ is less than $t_{0}$, and $H\left(t-t_{0}\right)=1$, when $t$ is greater than or equal to $t_{0}$. Note that $N$ in Equations (6)-(7) may be different depending on the number of hours used per segment. For this research, the glucose effectiveness, $p_{G}$, is held constant over two hour periods and the insulin sensitivity, $S_{I}$, varies every hour, creating piecewise constant time varying model parameters. Finally, the variation in Equations (6)-(7) can also be defined as linear or higher order, over these time periods for greater detail, at a cost of increasing the number of unknown parameters. Other parameters or different length time segments maybe used with no loss of generality.

The only unknown parameters in Equation (5) are $p_{G i}$ and $S_{I i}$ when Equations (6) and (7) are used. However, these are constant parameters such that after numerically integrating the data, Equation (5) can be written as a simple linear system in terms of these unknown values.

$$
\underline{A}\left\{\begin{array}{c}
p_{G i} \\
S_{I i}
\end{array}\right\}=\underline{b}
$$


where the number of equations for each time segment can be arbitrarily selected by integrating over different time segments. To ensure that the values obtained for $p_{G}$ and $S_{I}$ are within physiologically valid ranges, weighted constraints are placed on both parameters when solving Equation (8).

To compute the integrals in Equation (5) over different time segments, the total blood glucose profile, $G_{T}$, is approximated using simple linear interpolation between the data points, forming a piecewise linear curve $G_{T-a p p r o x}$. The error between the patient's real glucose level $G_{T-\text { real }}$ and the approximated curve $G_{T-a p p r o x}$ is defined:

$$
G_{T-\text { real }}(t)=G_{T-a p p r o x}(t)+\varepsilon(t)
$$

where the error term, $\varepsilon(t)$, should fall within the interval, $0 \leq|\varepsilon(t)| \leq \delta$, for small $\delta$, and $\delta$ is a measure of the theoretically best possible fit of the model to the data.

Using Equation (5), $G_{T-\text { real }}$ can be calculated in any given time interval $\left[t_{0}, t\right]$ with the constant parameters $p_{G i}$ and $S_{I i}$ as defined in Equations (6)-(7). 


$$
\begin{array}{r}
G_{T-\text { real }}(t)=G_{T-\text { real }}\left(t_{0}\right)-p_{G i} \int_{t_{0}}^{t}\left(G_{T-\text { real }}(t)-G_{E}\right) d t \\
-S_{I i} \int_{t_{0}}^{t} G_{T-\text { real }}(t) \bar{Q}(t) d t+\int_{t_{0}}^{t} P(t) d t \\
=G_{T-\text { approx }}\left(t_{0}\right)+p_{G i}\left(t-t_{0}\right) G_{E}-p_{G i} \int_{t_{0}}^{t} G_{T-\text { approx }}(t) d t \\
-S_{I i} \int_{t_{0}}^{t} G_{T-\text { approx }}(t) \bar{Q}(t) d t+\int_{t_{0}}^{t} P(t) d t+E(t)
\end{array}
$$

where the error term $E(t)$ arises from using the approximate piecewise linear blood glucose profile. The magnitude and order of $E(t)$ is defined:

$$
\begin{aligned}
|E(t)| & =\left|\varepsilon\left(t_{0}\right)-p_{G i} \int_{t_{0}}^{t} \varepsilon(t) d t-S_{I i} \int_{t_{0}}^{t} \varepsilon(t) \bar{Q}(t) d t\right| \\
& \leq\left|\varepsilon\left(t_{0}\right)\right|+p_{G i}\left|\int_{t_{0}}^{t} \varepsilon(t) d t\right|+S_{I i}\left|\int_{t_{0}}^{t} \varepsilon(t) \bar{Q}(t) d t\right| \\
& \leq \delta+p_{G i} \int_{t_{0}}^{t}|\varepsilon(t)| d t+S_{I i} \int_{t_{0}}^{t}|\varepsilon(t)| \bar{Q}(t) d t \\
& \leq \delta+p_{G i} \delta\left(t-t_{0}\right)+S_{I i} \delta \int_{t_{0}}^{t} \bar{Q}(t) d t \\
& =O(\delta)
\end{aligned}
$$

Note that even if the time period, $\left[t, t_{0}\right]$, and the integral, $\int_{t_{0}}^{t} \bar{Q}(t) d t$, are both large, the piecewise patient specific parameter terms, $p_{G i} \delta\left(t-t_{0}\right)$ and $S_{I i} \delta \int_{t_{0}}^{t} \bar{Q}(t) d t$, are small compared to the integral patient specific terms, $p_{G i} \int_{t_{0}}^{t} G_{T-\text { real }}(t) d t$ and 
$S_{I i} \int_{t_{0}}^{t} G_{T-\text { real }}(t) \bar{Q}(t) d t$, when it is assumed that the glucose level data is greater than 1 $\mathrm{mmol} / \mathrm{L}, G_{T-\text { real }}(t)>1 \mathrm{mmol} / \mathrm{L}$, which is always true for a living patient. The first two error terms in Equation (11) yield:

$$
\begin{gathered}
\left|\frac{p_{G i} \delta\left(t-t_{0}\right)}{p_{G i} \int_{t_{0}}^{t} G_{T-\text { real }}(t) d t \mid}\right|<\frac{\delta\left(t-t_{0}\right)}{\int_{t_{0}}^{t} 1 d t}=\delta \\
\left|\frac{S_{I i} \delta \int_{t_{0}}^{t} \bar{Q}(t) d t}{\mid S_{I i} \int_{t_{0}}^{t} G_{T-\text { real }}(t) \bar{Q}(t) d t}\right|<\frac{\delta \int_{t_{0}}^{t} \bar{Q}(t) d t}{\int_{t_{0}}^{t} \bar{Q}(t) d t}=\delta
\end{gathered}
$$

where $G_{T \text {-real }}(t)=1 \mathrm{mmol} / \mathrm{L}$ has been used to show the lower limit.

More specifically, $\delta$, as defined in Equation (9) measures how well the model captures the measured data. Thus, the fit is not affected by the linear approximation of the glucose data when using this integral formulation. Therefore, for a general time period $\left[t_{0}, t\right]$ this approximation of the glucose data can be used to represent the best fit, $G_{T-\text { real }}$, utilising integral functions, as in Equation (5), to identify the parameters defining the piecewise constant definitions for $p_{G}$ and $S_{I}$ from Equations (6)-(7) without introducing additional error.

The integrals in Equation (5) can be determined for several different time periods in a given data set to find $p_{G}$ and $S_{I}$. For example, when the length of the time period is chosen to be 120 minutes long, $\left[t_{0}, t_{0}+120\right]$, the fractional clearance of glucose, $p_{G}$, 
takes on one value, $p_{G 1}$, as defined here and the insulin sensitivity parameter, $S_{I}$, takes on two values, $S_{I 1}$ and $S_{I 2}$, giving three unknowns for this time period. To find the patient specific parameter values that give the best fit to the measured glucose data in this time interval, six equations are proposed with the generic form:

$$
G_{T-\text { approx }}\left(t_{0}+20 i\right)-G_{T-f i t}\left(t_{0}+20 i\right)=0, \quad i=1 \ldots 6
$$

To show the equations that result, the first and last of these equations, $i=1$ and $i=6$, are given:

$$
\begin{gathered}
G_{T-\text { approx }}\left(t_{0}+20\right)-G_{T-\text { approx }}\left(t_{0}\right)-20 p_{G_{1}} G_{E}+p_{G_{1}} \int_{t_{0}}^{t_{0}+20} G_{T-\text { approx }}(t) d t \\
+S_{I_{1}} \int_{t_{0}}^{t_{0}+20} G_{T-\text { approx }}(t) \bar{Q}(t) d t-\int_{t_{0}}^{t_{0}+20} P(t) d t=0 \\
G_{T-\text { approx }}\left(t_{0}+120\right)-G_{T-\text { approx }}\left(t_{0}\right)-120 p_{G_{1}} G_{E}+p_{G_{1}} \int_{t_{0}}^{t_{0}+120} G_{T-\text { approx }}(t) d t \\
+S_{I_{1}} \int_{t_{0}}^{t_{0}+60} G_{T-\text { approx }}(t) \bar{Q}(t) d t+S_{I_{2}} \int_{t_{0}+60}^{t_{0}+120} G_{T-\text { approx }}(t) \bar{Q}(t) d t-\int_{t_{0}}^{t_{0}+120} P(t) d t=0
\end{gathered}
$$

where the integrals in Equations (15) and (16) can be readily evaluated numerically. The unknowns in these equations are $p_{G 1}$, and $S_{I 1}$ and $S_{I 2}$. Therefore, for $i=1 \ldots 6$, Equation (14) defines a least squares system of six linear equations with three unknowns. The convex solution of these parameters consequently defines the timevarying profile for $p_{G}$ and $S_{I}$ for that time period. Finally, note that using integrals, instead of derivative based fitting methods, has the advantage of being robust to noise in the measured glucose data, effectively providing a low-pass filter in the summations involved in numerically integrating. 


\section{Patient Data and Selection Criteria}

For the long term data fitting and model verification, a random selection of 17 patients from a 201 patient data audit at Christchurch Hospital were selected [21]. Each patient record had a period greater than one day with intervals between measured data points of three hours or less. The data density of three hours was selected to ensure enough measurements to enable a good model evaluation. The entire length of stay was not always considered, as many patients only had a shorter period of data that fitted the criteria. This subset broadly represents the cross section of patients seen in the ICU, regarding medical condition, age, sex, APACHE II scores and mortality, as summarised in Table I. Type 1 and Type 2 diabetes is somewhat over-represented because these patients are often more frequently measured. Note that BMI is not typically recorded in most ICU's and was therefore not retrospectively available. Ethical consent was obtained from the Canterbury Ethics Committee for this research.

\section{RESULTS}

Four of the 17 resulting parameter identifications are shown in Figures 1-4. To further reduce the impact of noise and erroneous measurements, the fitted values of $p_{G}$ and $S_{I}$ were replaced by their smoothed, zero-phase 3-point moving average to produce the fitted model curve in each figure. The result is a less exact fit, but a smoother more physiological time-varying profile for these parameters. Figure 5 shows the fitting of patient 87 from Figure 2 without smoothing for comparison. 
Patient 1090 in Figure 1 had the lowest fitting error and standard deviation of the 17 patients considered, representing the best overall fit. Over the 1.6 days considered of the patient's 7-day stay, the insulin infused was constant at $1 \mathrm{U} / \mathrm{hr}$, and the feed, $P(t)$, was constant at $0.0247 \mathrm{mmol} /(\mathrm{L} \cdot \mathrm{min})$. Figure 1 also shows a relatively constant normoglycemic profile over this time with low variations in insulin sensitivity, $S_{I}$, showing that this patient was very stable. The parameter $p_{G}$ was effectively constant at $0.01 \mathrm{~min}^{-1}$ where $S_{I}$ shows some diurnal variation. These values for $p_{G}$ and $S_{I}$ are well within physiological ranges reported in the literature (e.g. $[17,18,21])$.

Patient 87 in Figure 2 had a total length of stay of 35 days, with sufficient data for model fitting over six consecutive days. Unlike patient 1090, the glucose level is much more variable, as is the resulting insulin sensitivity, $S_{I}$. The glucose effectiveness $p_{G}$, is stable over the time considered at $0.01 \mathrm{~min}^{-1}$, but does reach values of up to $0.015 \mathrm{~min}^{-1}$ on different days. These values are well within the physiological ranges reported in the literature (e.g. $[17,18,21])$, and show a change in the metabolic responses at these time periods. Over the six days, the insulin infusion and glucose feed varied significantly as well.

Patient 519 in Figure 3 had a total length of stay close to two weeks and shows the effectiveness of the model and integral fitting method as the model goes through every error bar. The parameter $p_{G}$ was again quasi-constant and $S_{I}$ varied significantly but, both were well within the physiological range. Patient 1025 in Figure 4 shows similar results over a smaller time period. 
Table II summarises the $p_{G}$ and $S_{I}$ values found for all the patients. The standard deviations for $p_{G}$ are all approximately 5-10 times smaller than the mean value indicating that $p_{G}$ remains relatively constant as seen in Figures 1-4. This result validates the use of 2 hour, or longer, constant values for $p_{G}$. Similarly, $S_{I}$ is much more variable in all four Figures and in Table II indicating the 1 hour, or shorter, window as appropriate. These results also match clinical expectations [13,22].

\section{A. Error Analysis:}

To quantify the error in data fitting, the "chi-square", $\chi^{2}$, quantity is calculated for the boundaries of the range of the glucose measurement error $(3.5-7 \%)$. This measure is the same criterion used to define an acceptable fit with the typically used non-linear recursive least squares (NRLS) method [9] which is non-convex for this problem. The expected value of $\chi^{2}$ is $v=N-M$, where $N$ is the number of measurements and $M=2$ the number of parameters identified. The results for each patient and the overall average values are given in Table III.

The average value of $v$ lies between $\chi^{2}{ }_{3.5}$ and $\chi^{2}{ }_{7}$, corresponding to a measurement error of $4.79 \%$, which is in the range of the reported sensor measurement error. The average verification interval was 3.1 days, with the longest at 12.3 days. The average fitting error over all patients was $4.31 \%$, with a range of $0.87-7.42 \%$. These values are at or within the experimental error, and match the expectations of other fitting methods.

\section{B. Model prediction:}

For use in control, it is not enough to capture dynamics by data fitting. The model must also be able to predict the behaviour and trends at least 1-2 hours ahead. A 
model that fits observed dynamics accurately and provides good predictions is well verified for clinical control applications. Ten patients were used to test the one-hour predictive ability of the identified model.

To make a forward prediction from a given point, the model fit from the data over the previous 8 hours was used. Note that predictions are insensitive to the length of time fitted prior to prediction, as long as it is greater than two hours or 3-4 measurements. The prediction is made by holding the current identified patient specific parameters, $p_{G}$ and $S_{I}$, and the equilibrium glucose level, $G_{E}$, constant over the next hour. The resulting model predicted value is compared to the actual data and the percentage absolute error, $e_{i}$, calculated. For patients where there was insufficient consecutive data every hour, the intermediate blood glucose targets were obtained via interpolation between data points. This process was repeated in moving 9-hour blocks, generating 13-76 predictions per patient. The results in Table IV show that one-hour predictions have an average absolute error of $2-11 \%$, which is close to the measurement error.

\section{Sensitivity Analysis}

A sensitivity analysis was done on the constant parameters $k, n, \alpha_{I}$ and $\alpha_{G}$ for patient 87 and variations in individual parameters of $-50,-10,+10$ and +50 percent. Table $\mathrm{V}$ shows the resulting percentage change in the mean and standard deviation of the fitted $S_{I}$ values. The parameter $p_{G}$ was not included as it was essentially constant and did not change more than $1 \%$. Variations of $k, n$ and $\alpha_{l}$ gave very little change to the identified $S_{I}$ but $\alpha_{G}$ gave a significant change as shown in Figure 6 and Table V. Note in Figure 6 that changes in $\alpha_{G}$ resulted in several data points being missed in days 4-6, 
unlike in Figure 2 where $\alpha_{G}$ is at its assumed value for Patient 87 . Hence, the $S_{I}$ values identified are not accurate and also result in the large errors in Table V. This result suggests $\alpha_{G}$ may need to be included in the identification process.

The results in Table $\mathrm{V}$ also show that $k, n$ and $\alpha_{l}$ can be fixed at the population values and errors will have little effect on the identified values of $S_{I}$ or $p_{G}$. Note that the standard deviation of $n$ is small compared to the mean showing that the $S_{I}$ curve is effectively scaled up or down. Figure 7 shows an example of this scaling.

All the results in the fitting suggest that $p_{G}$ is essentially constant, so tests were done holding $p_{G}=0.01$ constant throughout. Table VI shows the results for all the patients. The mean absolute errors in the modelled glucose values are given, as well as the standard deviation. The results show very similar errors suggesting that $p_{G}$ might also be held at a population value similar to $k, n$ and $\alpha_{I}$.

\section{Variations in Insulin Sensitivity:}

A number of researchers have explored the idea of variation in insulin sensitivity in ambulatory diabetic and normal individuals (e.g. $[13,23,24]$ ). To the authors' knowledge, little is known about variations of insulin sensitivity in critically ill patients. By using the insulin sensitivity profiles from long-term fitting of the 17 patients considered, any cyclical variations may be observed. To find the period of a cycle in these patients, the average oscillation period of insulin sensitivity, $S_{I}$, can be determined by finding the inflection points in the insulin sensitivity curve, $S_{I}(t)$, where the second derivative is zero. The range in cycle length is $0.23-0.54$ days (5.5-13.0 
hours). Taking into account the number of cycles for each patient, the average period over all the patients is 0.45 days ( 10.8 hours) which is very close to half a day, suggesting a potential half day variation cycle for insulin sensitivity in critically ill patients.

\section{E. Comparison to NRLS:}

To accurately test the performance of the integral method versus the NRLS method the integrals were replaced by an ODE solver that solves the differential equations over the entire period. The results were used to implement the NRLS method in MATLAB on a Pentium 4 desktop with $3.0 \mathrm{GHz}$ CPU and 1.0 GMb of RAM. The known physiological ranges of $p_{G}$ and $S_{I}$ were also included in the optimization. Thus, the two approaches were made as similar as possible. The results for patient 87 with a 6.4 day stay are given in Table VII.

Table VII shows the integral method is approximately 1000 times faster than the nonlinear method and gives slightly more accurate results for this larger simulation over a very long time period. Note that the non-linear method found a local minima that was not the global minimum. To find the global minimum the method would have to use other starting points none of which are guaranteed to find the global minimum, significantly increasing computational time. No other patients were simulated using NRLS because of the CPU times involved. The large CPU time is due to the very long time periods examined compared to other typical applications such as clamp tests. As a result, the ODE solvers required significantly more computation given the small time steps required. Hence, NRLS is not as suitable for use over such long time periods, even if broken into smaller segments for solution. 


\section{DISCUSSION}

The model presented is physiologically justified and utilizes the concept of a remote compartment for insulin storage to account for the delay between insulin secretion, or infusion, and utilization. The minimal model of Bergman et al [10] was designed to model metabolic physiology and simulate basic glucose dynamics in vivo, and to be sufficiently simple to estimate patient specific parameters using data from the steady state a frequently-sampled intravenous glucose tolerance test (FSIGTT). Its primary focus was on steady state dynamics for the estimation of insulin sensitivity, and not for transient dynamic cases as it neglects the time-varying utilisation of insulin and glucose removal. This effect as well as saturation is accounted for in the model presented.

The fitting method proves very effective in reducing a typically non-linear, nonconvex optimization problem to a simple convex, linear system. All fitted values for $p_{G}$ and $S_{I}$ are within physiologically valid ranges reported in the literature. The integral method is shown to be very fast and has a very significant computational speed advantage over the current non-linear approach as integrals are computationally easier than repeated simulations over several days. Finally, note that optimized code in $\mathrm{C} / \mathrm{C}^{++}$or FORTRAN as well as application over shorter tests (e.g. 2 hours) would reduce the computational differences. 
Thus the integral approach makes it feasible to modify additional parameters like $k$ and $\alpha_{G}$ through a range of physiological ranges without slowing the algorithm down so much that it may affect real time applications. For example, $\alpha_{G}$ could be fixed at a number of values and the fitting method applied for each value. The non-linear optimization method is severely restricted in this regard because of the significantly slower identification of $S_{I}$ and $p_{G}$. Hence, the non-linear optimization is a less suitable way for model testing and identification over long time periods.

Glucose effectiveness, $p_{G}$, remained nearly constant throughout all patients and tests, suggesting it can be held constant. This is an interesting result and could be used in real time trials if it was continually the case. The parameter $S_{I}$ showed much greater variation. Some of this variability is due to the highly variable condition of the critical care patients, which can induce large variation in the level of counter-regulatory hormones present and hence in insulin sensitivity. In addition, drug therapies such as $\beta$-blockers or vaso-dilators can also have an impact on insulin sensitivity.

The average fitting error is in the range of the sensor error and matches the expected $\chi^{2}$ value. The low long-term-fit error values validate the models ability to capture the highly variable dynamics of hyperglycemic ICU patients over long periods. Note that prior to three-point smoothing to reduce the effects of measurement noise, the identified fits are within $1 \%$ error. However, the variations observed for the patient specific parameters are potentially non-physiological, although they are within reported physiological ranges. 
Measurements for these 17 subjects were primarily taken 2-3 hours apart, which can lead to dynamics being missed that could be captured with more frequent measurement. This low measurement frequency may be one reason that the parameters $\left(V_{I}=12 \mathrm{~L}, n=0.16 \mathrm{~min}^{-1}, k=0.0099 \mathrm{~min}^{-1}, \alpha_{I}=0.0017 \mathrm{~L} / \mathrm{mU}\right)$ were able to be held constant while still obtaining very good fits. The sensitivity analysis supports this result as changing the parameters from their population values did not significantly affect the fits and the trends of the primary parameters $p_{G}$ and $S_{I}$.

Controlled critical care insulin infusion trials that measure blood glucose every 30 minutes have shown the need to allow slight variation of $\alpha_{G}$ to obtain the same accuracy reported in this study [25] with higher measurement density. This result also matches the sensitivity analysis results here, which indicate that $\alpha_{G}$ may vary over time. One possible cause could be the more variable changes in cortisol-binding globulin and insulin-like growth hormone levels in critical care patients both of which impact insulin action.

The forward prediction of glucose values for a period of one hour ahead were within $2-11 \%$ of the measured value, further validating the $p_{G}$ and $S_{I}$ values obtained. This error is also within the clinically acceptable " $\mathrm{A}$ " range for glycaemic control at $\pm 20 \%$ using the Clarke Error Grid. The $2-11 \%$ range is also within or near the sensor error. These results show the potential for the use of this model for clinical control. More specifically, given the patient specific parameters at any point in time the amount of insulin can be directly calculated to reduce a patient's glucose level to a target level in the next hour. This process could be continued every hour so that a 
patient's glucose level is tightly controlled over their entire stay at the ICU with minimal variation.

Note that holding $p_{G}$ and $S_{I}$ constant during the prediction interval is a conservative choice, as it ignores the prior changes in value where some other methods use the prior trends. Analysis of prior trends and potential hourly variation in these parameters is a topic of current research. Finally, the half-day cycle of variation seen in insulin sensitivity is similar to the diurnal variations noted in diabetic individuals (e.g. [23]).

\section{CONCLUSIONS}

The integral based parameter identification method enables the time varying model parameters $S_{I}$ and $p_{G}$ to be rapidly identified with minimal computation. It provides more accurate results and significant computational speed gains versus current nonlinear methods for model identification using long-term data. It is therefore capable of analyzing large numbers of patients in a short time which is important in model testing and refinement to capture all observed patient behaviour. Furthermore, it is easily implemented using a simple programming language like MATLAB on a standard desktop computer.

The identified model was able to capture the dynamics in the measured data to within the $7 \%$ sensor error, and was tested for time periods up to two weeks. The model provides a prediction for one hour ahead with absolute errors between $2-11 \%$, which is acceptable for control applications in a clinical setting. Further applications are 
studying the changes of insulin sensitivity and glucose effectiveness of patients over long periods and comparing the results to those measured by independent tests like the insulin or glycemic clamp. However the main application for this model and integral based identification method is the real-time automated control of glucose levels in ICU patients and similar medical decision support systems.

\section{REFERENCES}

[1] S.E. Capes, D. Hunt, K. Malmberg, H.C. Gerstein, Stress Hyperglycaemia and Increased Risk of Death after Myocardial Infarction in Patients with and without Diabetes: A Systematic Overview, Lancet, 355 (9206) (2000) 773778.

[2] G. Van den Berghe, P. Wouters, F. Weekers, C. Verwaest, F. Bruyninckx, M. Schetz, D. Vlasselaers, P. Ferdinande, P. Lauwers, R. Bouillon, Intensive Insulin Therapy in the Critically Ill Patients, N Engl J Med. 345 (19) (2001) 1359-1367.

[3] B.A. Mizock, Alterations in Fuel Metabolism in Critical Illness: Hyperglycaemia, Best Pract Res Clin Endocrinol Metab. 15 (4) (2001) 533551.

[4] K.C. McCowen, A. Malhotra, B.R. Bistrian, Stress-Induced Hyperglycemia, Crit Care Clin, 17 (1) (2001) 107-124.

[5] B.R. Bistrian. Hyperglycemia and Infection: Which Is the Chicken and Which Is the Egg? JPEN J Parenter Enteral Nutr. 25 (4): (2001) 180-181.

[6] J.S. Krinsley. Association between Hyperglycemia and Increased Hospital Mortality in a Heterogeneous Population of Critically Ill Patients, Mayo Clin Proc. 78 (12) (2003) 1471-1478.

[7] C.V. Doran, J.G. Chase, G.M. Shaw, K.T. Moorhead, N.H. Hudson, Automated Insulin Infusion Trials in the ICU, Diabetes Technology and Therapeutics. 6 (2) (2004).

[8] A.M. Albisser, B.S. Leibel, T.G. Ewart, Z. Davidovac, C.K. Botz, W. Zingg, H. Schipper, R. Gander, Clinical Control of Diabetes by the Artificial Pancreas, Diabetes. 23 (5) (1974) 397-404.

[9] R. Hovorka, P. Vicini, in E. Carson, C. Cobelli (Editors), Modelling Methodology for Physiology and Medicine, Academic Press, London, 2001.

[10] R.N. Bergman, D.T. Finegood, M. Ader, Assessment of Insulin Sensitivity in Vivo, Endocr Rev. 6 (1) (1985) 45-86.

[11] C. Cobelli, A. Caumo, M. Omenetto, Minimal Model SG Overestimation and SI Underestimation: Improved Accuracy by a Bayesian Two-Compartment Model, Am J Physiol. 277 (3 Pt 1) (1999) E481-488.

[12] K. Turnheim, W.K. Waldhausl, Essentials of Insulin Pharmacokinetics, Wien Klin Wochenschr. 100 (3) (1988) 65-72.

[13] R. Hovorka, V. Canonico, L.J. Chassin, M. Massi-Benedetti, M. O. Federici, T.R. Pieber, H.C. Schaller, L. Schaupp, T. Vering, M. E. Wilinska, Control of glucose in type 1 diabetes with subcutaneous insulin infusion: Non-linear 
model predictive control with Bayesian parameter estimation, World Congress on Biomedical Engineering and Medical Physics, Sydney, Australia, 2003.

[14] E.A. McGuire, J.D. Tobin, M. Berman, R. Andres, Kinetics of Native Insulin in Diabetic, Obese, and Aged Men, Diabetes. 28 (2) (1979) 110-120.

[15] P.H. Sonksen, C.V. Tompkins, M.C. Srivastava, J.D. Nabarro, A Comparative Study on the Metabolism of Human Insulin and Porcine Proinsulin in Man, Clin Sci Mol Med. 45 (5) (1973) 633-654.

[16] B. Thorsteinsson, Kinetic Models for Insulin Disappearance from Plasma in Man, Dan Med Bull. 37 (2) (1990) 143-153.

[17] R.L. Prigeon, M.E. Roder, D. Porte, Jr., S.E. Kahn, The Effect of Insulin Dose on the Measurement of Insulin Sensitivity by the Minimal Model Technique. Evidence for Saturable Insulin Transport in Humans, J Clin Invest. 97 (2) (1996) 501-507.

[18] A. Natali, A. Gastaldelli, S. Camastra, A.M. Sironi, E. Toschi, A. Masoni, E. Ferrannini, A. Mari, Dose-Response Characteristics of Insulin Action on Glucose Metabolism: A Non-Steady-State Approach, Am J Physiol Endocrinol Metab. 278 (5) (2000) E794-801.

[19] R.A. DeFronzo, J.D. Tobin, R. Andres, Glucose Clamp Technique: A Method for Quantifying Insulin Secretion and Resistance, Am J Physiol. 237 (3) (1979) E214-223.

[20] P. Vicini, A. Caumo, C. Cobelli, The Hot IVGTT Two-Compartment Minimal Model: Indexes of Glucose Effectiveness and Insulin Sensitivity, Am J Physiol. 273 (5 Pt 1) (1997) E1024-1032.

[21] C.V. Doran, Modelling and Control of Hyperglycaemia in Critical Care Patients, Masters of Engineering Thesis, Mechanical Engineering, University of Canterbury, Christchurch, New Zealand (2004).

[22] C. McDonald, A. Dunaif, and D.T. Finegood, Minimal-Model Estimates of Insulin Sensitivity Are Insensitive to Errors in Glucose Effectiveness, J Clin Endocrinol Metab. 85 (7) (2000) 2504-2508.

[23] M. P. Arasaradnam, L. Morgan, J. Wright, R. Gama, Diurnal Variation in Lipoprotein Lipase Activity, Ann Clin Biochem. 39 (Pt 2) (2002) 136-139.

[24] J. Radziuk, Insulin Sensitivity and Its Measurement: Structural Commonalities among the Methods, J Clin Endocrinol Metab. 85 (12) (2000) 4426-4433.

[25] J.G. Chase, G.M. Shaw, J.Lin, C.V. Doran, C.E. Hann, M.B. Robertson, P.M. Brown, T. Lotz, G.C. Wake, and B. Broughton, Adaptive Bolus-Based SetPoint Regulation of Hyperglycemia in Critical Care, Proc 26 th International Conf of IEEE Engineering in Med and Biology Society (EMBS 2004), San Francisco, CA, Sept 1-5 , (2004) 3463-3466. 

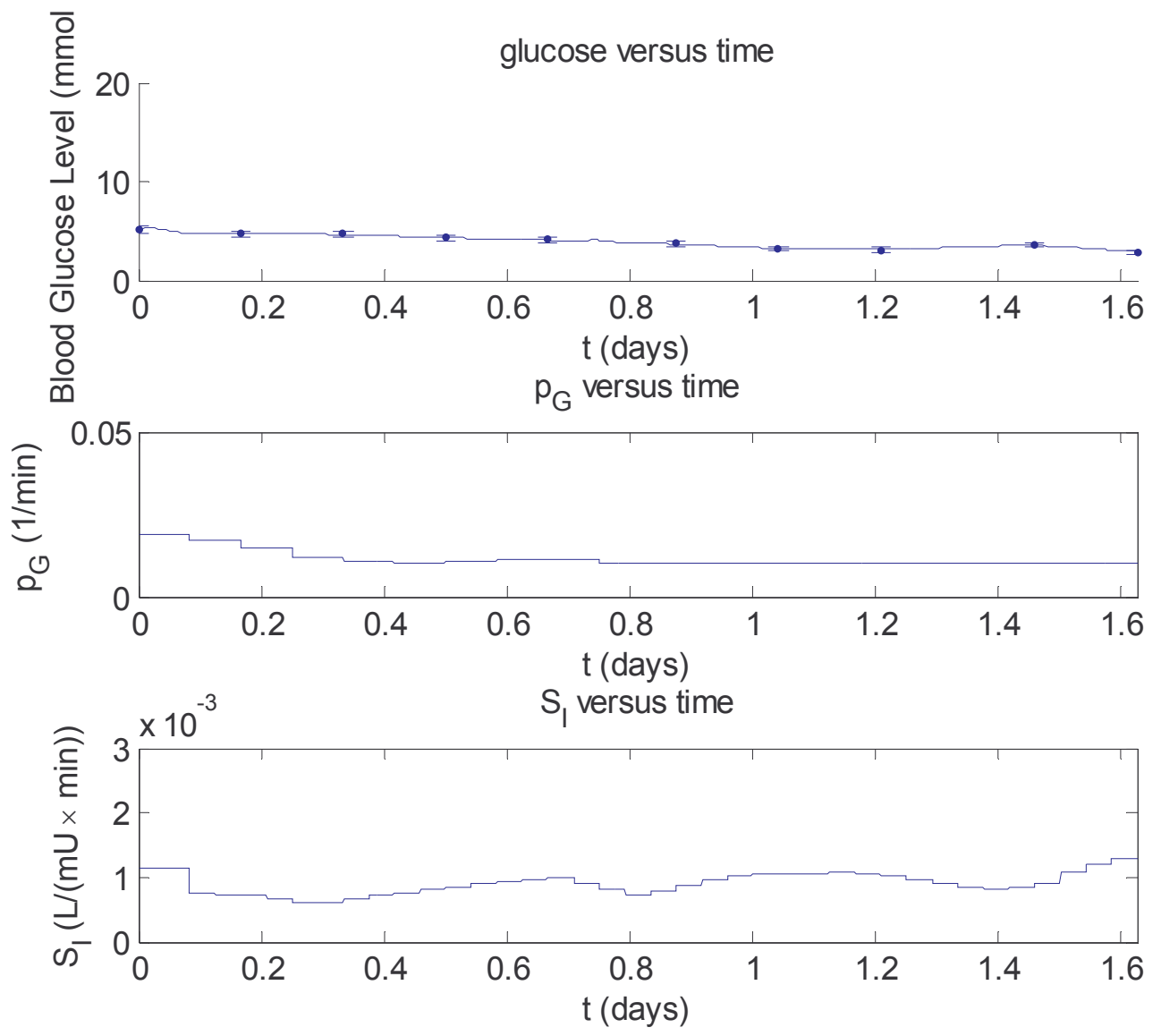

Figure 1: Patient 1090 blood glucose data fit (top), corresponding glucose

effectiveness $p_{G}$ (middle) and corresponding insulin sensitivity parameter $S_{I}$ (bottom). 

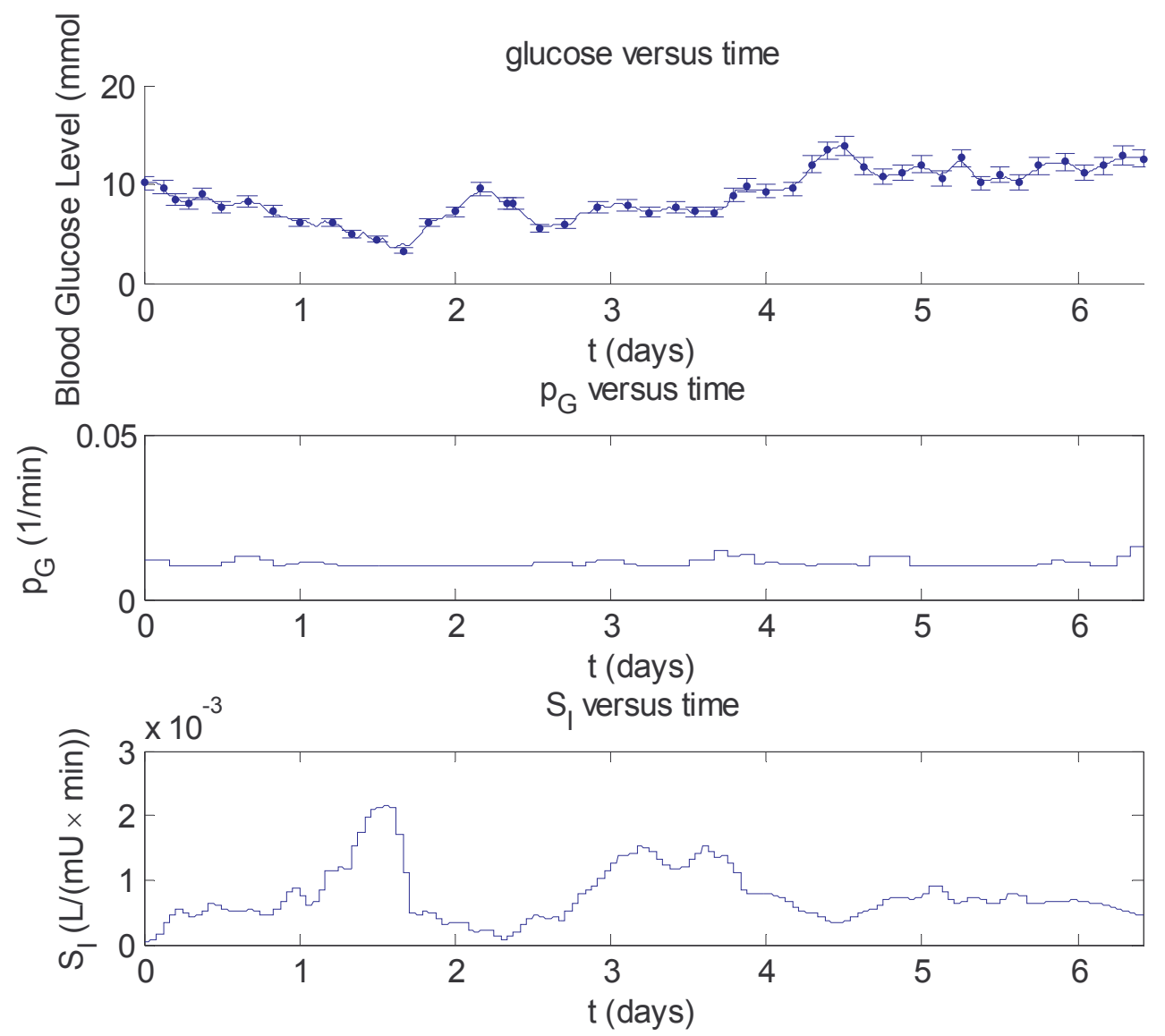

Figure 2: Patient 87 blood glucose data fit (top), corresponding glucose effectiveness $p_{G}$ (middle) and corresponding insulin sensitivity parameter $S_{I}$ (bottom). 

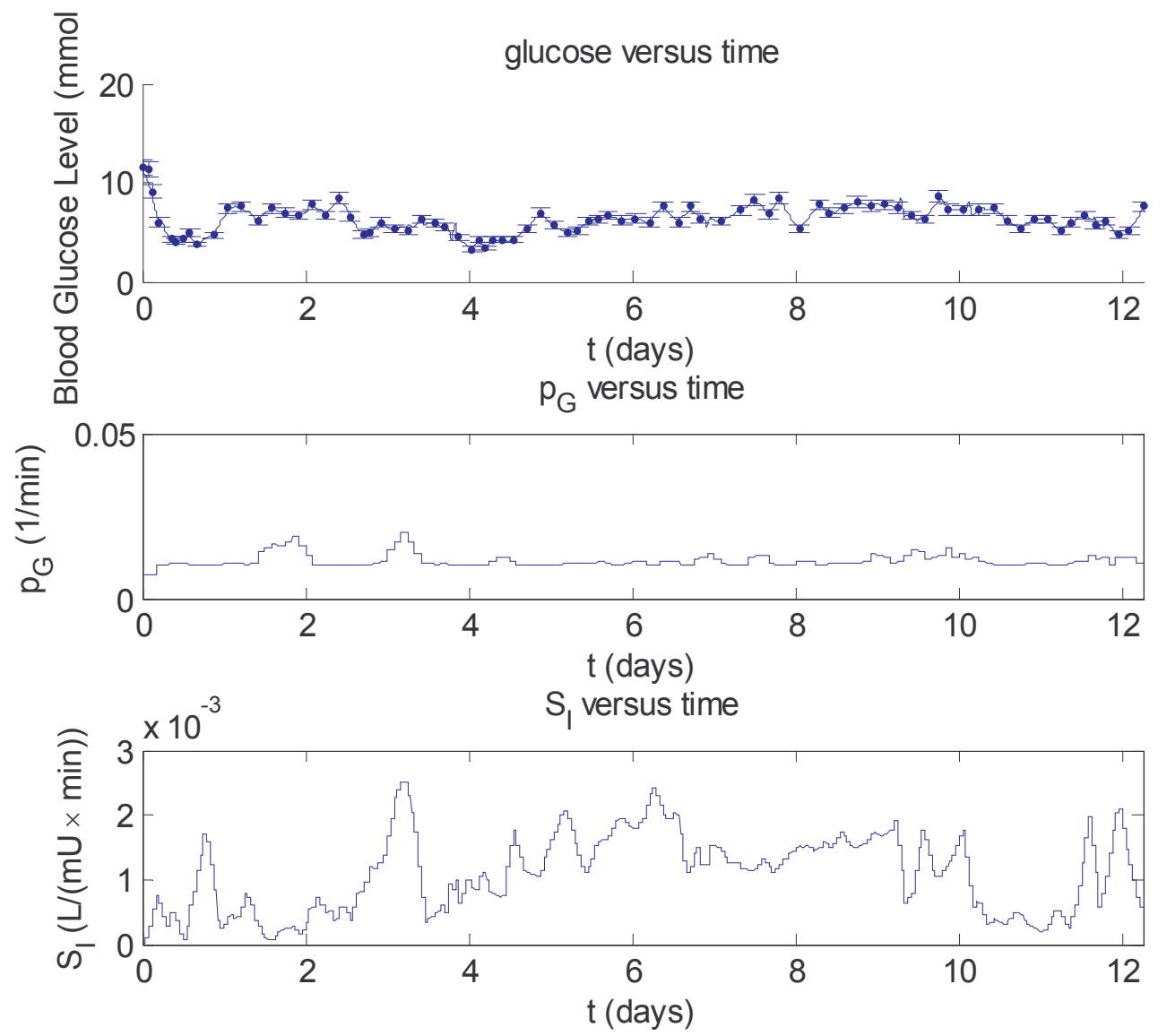

Figure 3: Patient 519 blood glucose data fit (top), corresponding glucose effectiveness $p_{G}$ (middle) and corresponding insulin sensitivity parameter $S_{I}$ (bottom). 

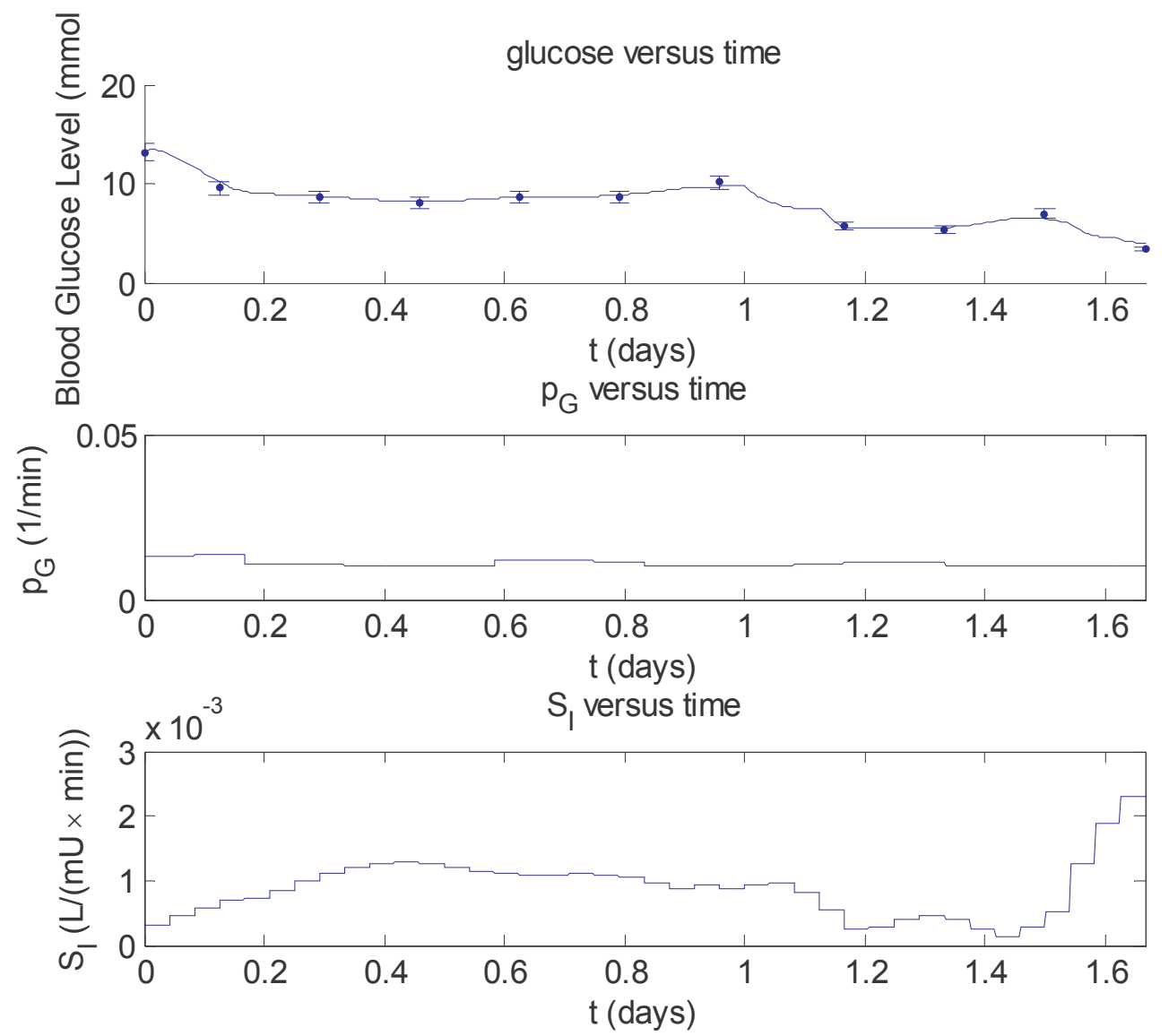

Figure 4: Patient 1025 blood glucose data fit (top), corresponding glucose effectiveness $p_{G}$ (middle) and corresponding insulin sensitivity parameter $S_{I}$ (bottom). 

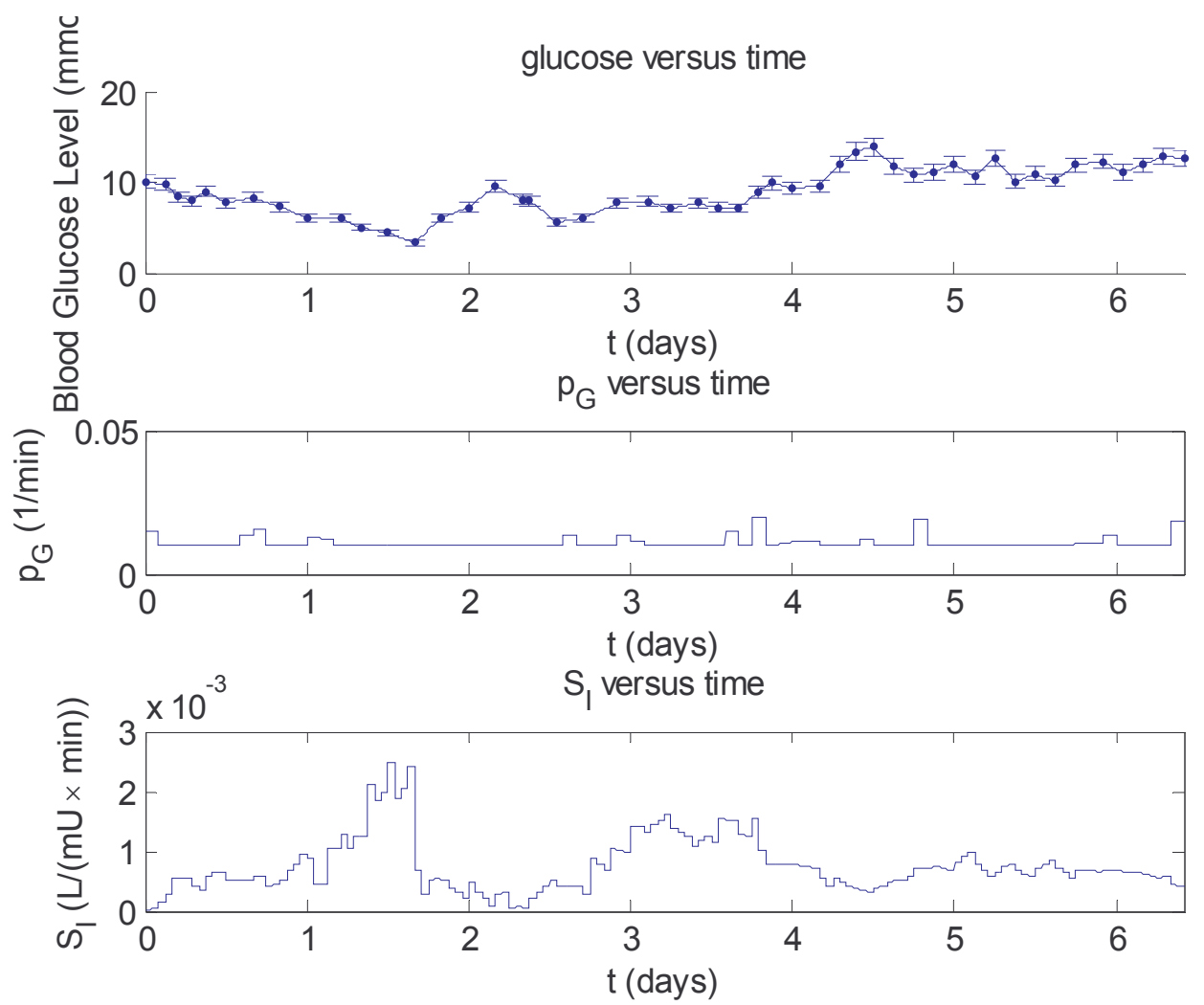

Figure 5: Fitted curve without smoothing $S_{I}$ for patient 87 of Figure 2. 

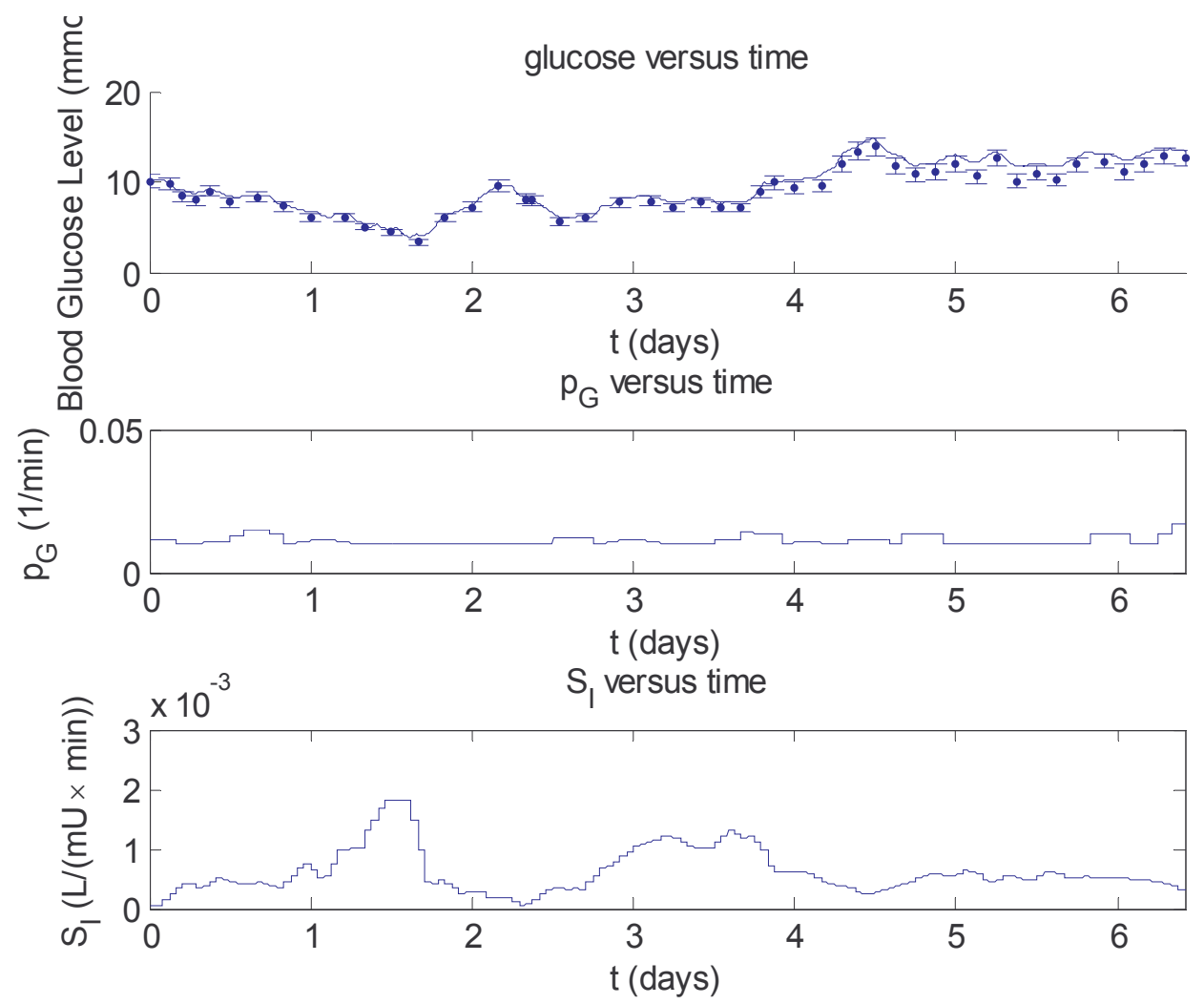

Figure 6: The effect of changing $\alpha_{G}\left(\alpha_{G}=1 / 25-0.5 \times 1 / 25,50 \%\right.$ error) for patient87. 

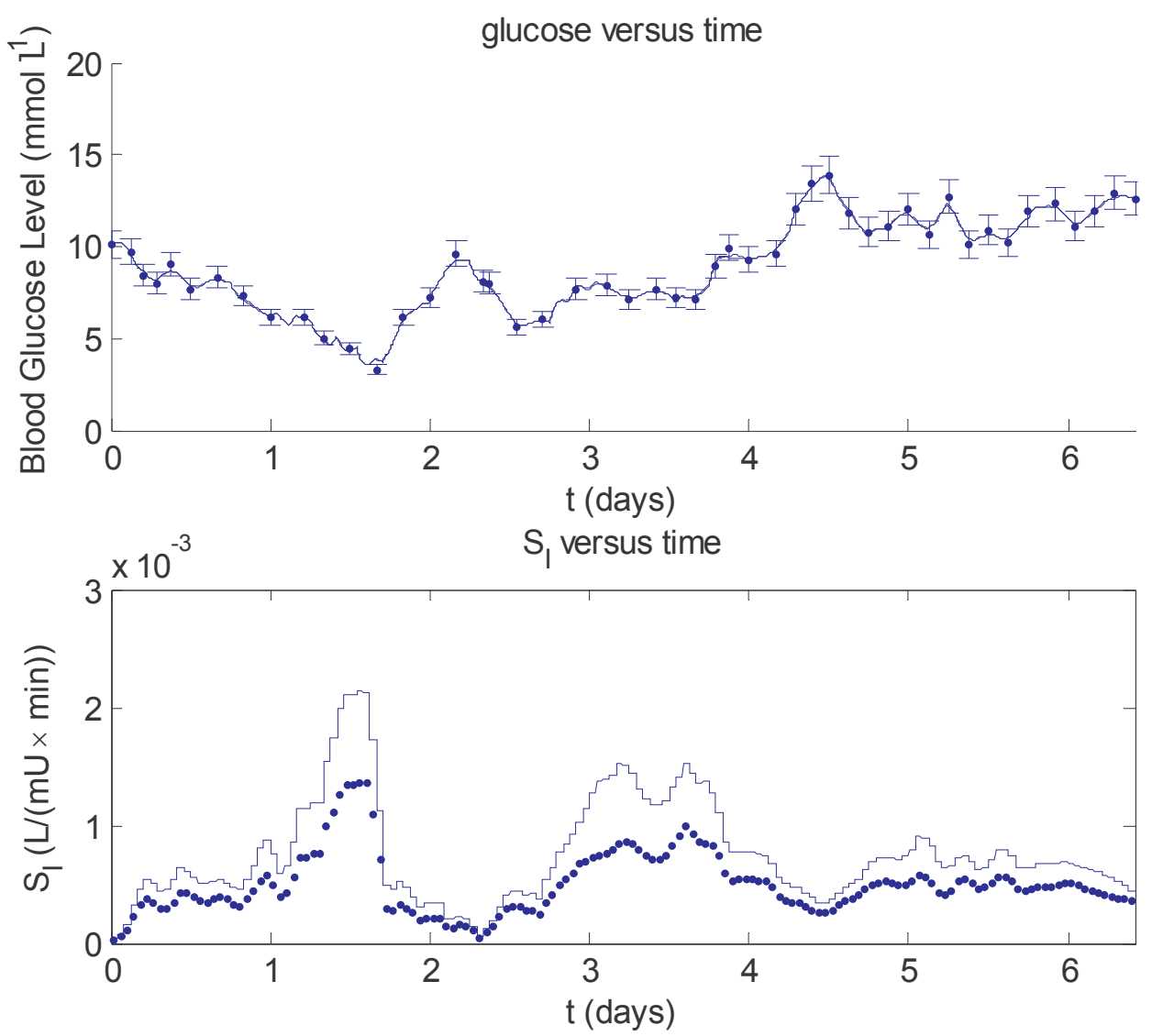

Figure 7: The effect of changing $n$ on patient87. The dotted curve of $S_{I}$ corresponds to $n=0.16-0.5 \times 0.16$ and the solid curve of $S_{I}$ corresponds to $n=0.16$ as also shown in Figure 2. The blood glucose curves are essentially overlaid. 
TABLE I: PATIENTS SELECTED FOR LONG-TERM DATA FITTING

\begin{tabular}{|c|c|c|c|c|c|c|}
\hline $\begin{array}{l}\text { Patient } \\
\text { Number }\end{array}$ & $\begin{array}{l}\text { Medical } \\
\text { Subgroup }\end{array}$ & $\begin{array}{l}\text { APACHE } \\
\text { II Score }\end{array}$ & Age & Sex & Mortality & Diabetes \\
\hline 24 & $\begin{array}{c}\text { Other } \\
\text { Medical }\end{array}$ & 25 & 47 & M & $\mathrm{Y}$ & Type 1 \\
\hline 87 & $\begin{array}{c}\text { Other } \\
\text { Medical }\end{array}$ & 26 & 62 & $\mathrm{~F}$ & & \\
\hline 130 & Trauma & 11 & 21 & $\mathrm{M}$ & & Type 1 \\
\hline 229 & Cardiac & 15 & 73 & $\mathrm{~F}$ & & \\
\hline 289 & Cardiac & 18 & 70 & $\mathrm{M}$ & & \\
\hline 468 & $\begin{array}{l}\text { General } \\
\text { Surgical }\end{array}$ & 32 & 76 & M & & \\
\hline 484 & $\begin{array}{c}\text { Other } \\
\text { Medical }\end{array}$ & 34 & 30 & $\mathrm{~F}$ & & \\
\hline 486 & $\begin{array}{l}\text { General } \\
\text { Surgical }\end{array}$ & 22 & 76 & $\mathrm{~F}$ & & Type 2 \\
\hline 519 & $\begin{array}{l}\text { General } \\
\text { Surgical }\end{array}$ & 29 & 69 & M & & Type 2 \\
\hline 554 & $\begin{array}{c}\text { Other } \\
\text { Medical }\end{array}$ & 26 & 20 & $\mathrm{~F}$ & & Type 1 \\
\hline 666 & Cardiac & 8 & 44 & $\mathrm{~F}$ & & Type 2 \\
\hline 847 & $\begin{array}{c}\text { Other } \\
\text { Medical }\end{array}$ & 17 & 67 & $\mathrm{~F}$ & & \\
\hline 1016 & $\begin{array}{l}\text { General } \\
\text { Surgical }\end{array}$ & 20 & 37 & $\mathrm{~F}$ & & Type 2 \\
\hline 1025 & Pulmonary & 36 & 48 & $\mathrm{M}$ & & Type 2 \\
\hline 1090 & $\begin{array}{l}\text { General } \\
\text { Surgical }\end{array}$ & Unknown & 37 & $\mathrm{~F}$ & & \\
\hline 1099 & Pulmonary & Unknown & 24 & $\mathrm{M}$ & $\mathrm{Y}$ & \\
\hline 1125 & $\begin{array}{c}\text { Other } \\
\text { Medical }\end{array}$ & Unknown & 72 & $\mathrm{~F}$ & $\mathrm{Y}$ & \\
\hline
\end{tabular}


TABLE II: FITTED PATIENTS PARAMETERS

\begin{tabular}{|c|c|c|c|c|}
\hline \multirow{2}{*}{$\begin{array}{c}\text { Patient } \\
\text { Number }\end{array}$} & \multicolumn{2}{|c|}{$\boldsymbol{p}_{\boldsymbol{G}}\left(\mathrm{min}^{-\mathbf{1}}\right)$} & \multicolumn{2}{c|}{$\boldsymbol{S}_{\boldsymbol{I}}\left(\mathrm{mU} \cdot \mathbf{L}^{-\mathbf{1}} \cdot \mathbf{m i n}^{-\mathbf{1}}\right)$} \\
\cline { 2 - 5 } & Mean & \multicolumn{1}{c|}{ Std } & \multicolumn{1}{c|}{ Mean } & \multicolumn{1}{c|}{ Std } \\
\hline 24 & 0.0101 & 0.0002 & 0.00048 & 0.00016 \\
\hline 87 & 0.0109 & 0.0013 & 0.00076 & 0.00044 \\
\hline 130 & 0.0106 & 0.0010 & 0.00045 & 0.00028 \\
\hline 229 & 0.0111 & 0.0014 & 0.00110 & 0.00060 \\
\hline 289 & 0.0100 & 0.0010 & 0.00110 & 0.00040 \\
\hline 468 & 0.0108 & 0.0008 & 0.00047 & 0.00024 \\
\hline 484 & 0.0108 & 0.0011 & 0.00049 & 0.00014 \\
\hline 486 & 0.0107 & 0.0010 & 0.00052 & 0.00032 \\
\hline 519 & 0.0114 & 0.0019 & 0.00110 & 0.00060 \\
\hline 554 & 0.0107 & 0.0012 & 0.00084 & 0.00054 \\
\hline 666 & 0.0101 & 0.0002 & 0.00032 & 0.00017 \\
\hline 847 & 0.0114 & 0.0013 & 0.00150 & 0.00060 \\
\hline 1016 & 0.0112 & 0.0015 & 0.00042 & 0.00019 \\
\hline 1025 & 0.0109 & 0.0011 & 0.00088 & 0.00045 \\
\hline 1090 & 0.0114 & 0.0026 & 0.00091 & 0.00017 \\
\hline 1099 & 0.0123 & 0.0019 & 0.00140 & 0.00040 \\
\hline 1125 & 0.0101 & 0.0014 & 0.00064 & 0.00037 \\
\hline
\end{tabular}


TABLE III: ERRORS IN PARAMETER IDENTIFICATION AND DATA FITTING $\begin{array}{lllll}\text { Patient Number } & \text { Time interval (days) } & \chi^{2}{ }_{3.5} & \chi^{2}{ }_{7} & v\end{array}$

\begin{tabular}{|c|c|c|c|c|}
\hline 24 & 2.0 & 57.82 & 14.46 & 29 \\
\hline 87 & 6.4 & 21.73 & 5.43 & 45 \\
\hline 130 & 1.4 & 142.20 & 35.55 & 28 \\
\hline 229 & 10.0 & 213.64 & 53.41 & 75 \\
\hline 289 & 1.7 & 49.52 & 12.38 & 10 \\
\hline 468 & 1.7 & 13.13 & 3.28 & 14 \\
\hline 484 & 1.7 & 8.88 & 2.22 & 15 \\
\hline 486 & 1.6 & 12.30 & 3.076 & 13 \\
\hline 519 & 12.3 & 62.73 & 15.68 & 79 \\
\hline 554 & 2.3 & 87.42 & 21.85 & 37 \\
\hline 666 & 1.6 & 15.48 & 3.87 & 12 \\
\hline 847 & 1.5 & 19.67 & 4.92 & 9 \\
\hline 1016 & 1.6 & 16.55 & 4.14 & 10 \\
\hline 1025 & 1.7 & 20.74 & 5.19 & 12 \\
\hline 1090 & 1.6 & 0.78 & 0.19 & 7 \\
\hline 1099 & 1.6 & 23.44 & 5.86 & 11 \\
\hline 1125 & 1.7 & 10.64 & 2.66 & 8 \\
\hline Average & 3.1 & 45.69 & 11.42 & 24.35 \\
\hline
\end{tabular}


TABLE IV: ONE HOUR MODEL PREDICTION ERRORS

\begin{tabular}{cccc}
\hline Patient & No. of predictions & $\begin{array}{c}\text { Average prediction } \\
\text { error } \boldsymbol{e} \mathbf{\%}]\end{array}$ & $\begin{array}{c}\text { Error standard deviation } \\
{[\mathbf{\%}]}\end{array}$ \\
\hline 24 & 22 & 5.86 & 4.00 \\
\hline 87 & 41 & 4.71 & 5.21 \\
\hline 130 & 18 & 10.12 & 9.55 \\
\hline 519 & 76 & 5.25 & 5.98 \\
\hline 554 & 24 & 10.90 & 8.89 \\
\hline 666 & 13 & 4.66 & 3.01 \\
\hline 1016 & 13 & 7.01 & 6.27 \\
\hline 1025 & 14 & 5.09 & 4.54 \\
\hline 1090 & 13 & 1.86 & 0.87 \\
\hline 1125 & 14 & 6.83 & 4.78 \\
\hline
\end{tabular}


TABLE V: SENSITIVITY ANALYSIS ON $S_{I}$ (expressed in $\%$ change in $S_{I}$ ) FOR PATIENT87

\begin{tabular}{|c|c|c|c|c|c|c|c|c|}
\hline \multirow{3}{*}{ Parameter } & \multicolumn{8}{|c|}{$\%$ change from population value used } \\
\hline & \multicolumn{2}{|c|}{-10} & \multicolumn{2}{|c|}{+10} & \multicolumn{2}{|c|}{-50} & \multicolumn{2}{|c|}{$+\mathbf{5 0}$} \\
\hline & mean & std & mean & std & mean & std & mean & std \\
\hline$k$ & 2.6 & 3.3 & 0.9 & 2.0 & 4.5 & 3.7 & 2.5 & 3.3 \\
\hline$n$ & 6.9 & 2.6 & 6.0 & 1.5 & 32.8 & 4.9 & 30.0 & 8.9 \\
\hline$\alpha_{I}$ & 0.2 & 0.2 & 3.1 & 4.7 & 1.2 & 1.8 & 4.1 & 4.7 \\
\hline$\alpha_{G}$ & 6.6 & 5.1 & 4.3 & 2.2 & 19.3 & 4.6 & 17.6 & 7.2 \\
\hline
\end{tabular}


TABLE VI: EFFECT ON FITTING ERROR BY HOLDING $p_{G}$ CONSTANT

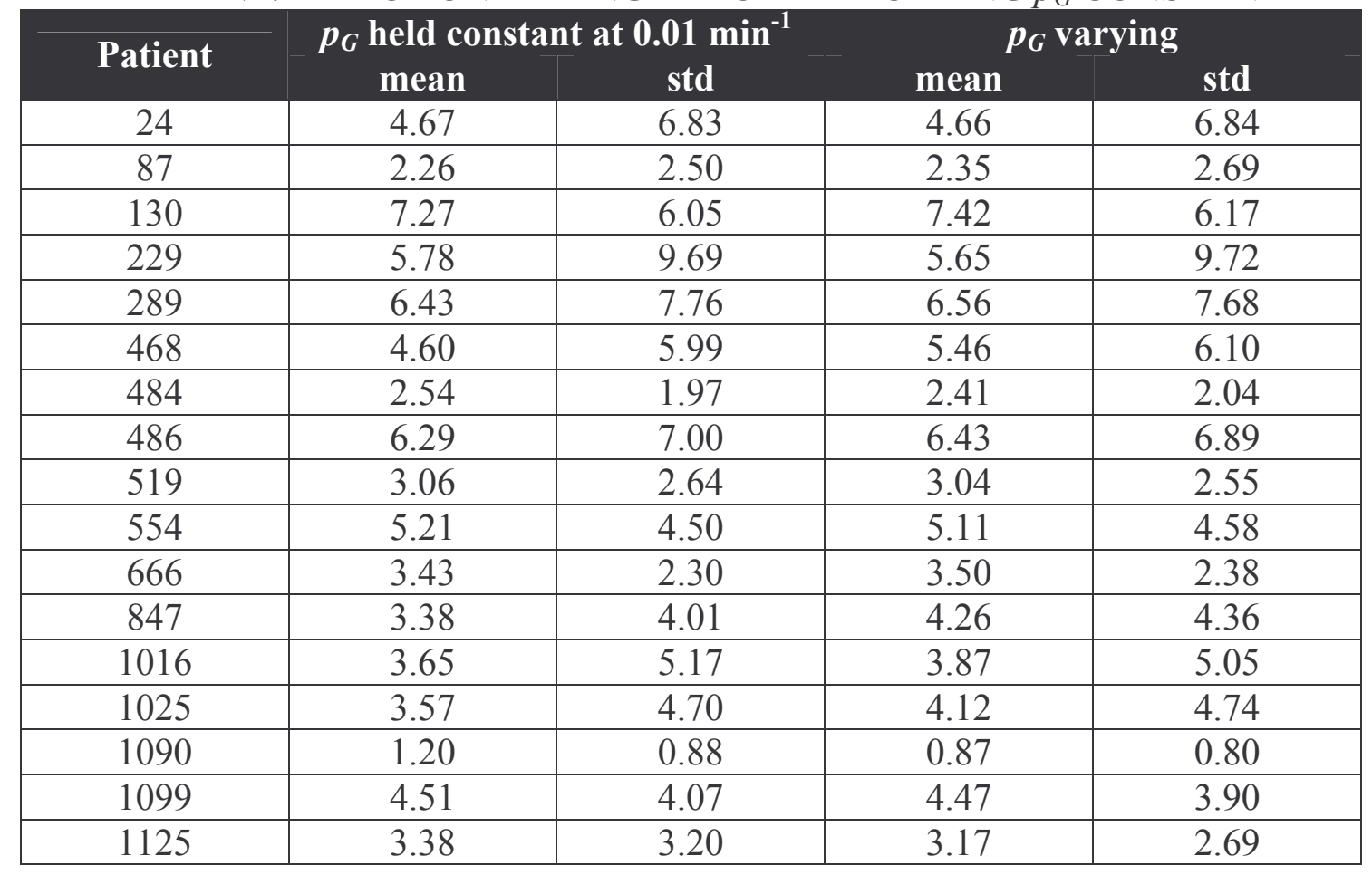


TABLE VII: COMPARISON BETWEEN INTEGRAL AND NON-LINEAR METHODS

\begin{tabular}{|c|c|c|c|c|}
\hline \multirow{2}{*}{ Patient 87} & \multirow{2}{*}{ CPU Time } & \multicolumn{2}{|c|}{ Relative \% Error } & \multirow{2}{*}{$\begin{array}{l}\text { Number of } \\
\text { error bars } \\
\text { missed }\end{array}$} \\
\hline & & mean & std & \\
\hline Integral Method & $\sim 30$ seconds & 2.35 & 2.69 & 1 \\
\hline Non-linear Method & $\sim 8$ hours & 4.68 & 5.52 & 10 \\
\hline
\end{tabular}

